\section{Different Radionuclides in DOTA-EB-TATE Effect Different Uptake in Somatostatin Receptor- Positive HEK293 Cells}

TO THE EDITOR: Tian et al. developed a trifunctional ligand with an albumin-binding component (Evans blue), which features a longer-circulation half-life as compared with an unmodified DOTA conjugate. It has been shown in a preclinical model that the prolonged circulation improved the accumulation of this tracer in somatostatin receptor (SSTR)-positive tumors (1). In this study, bioimaging was performed by ${ }^{86}$ Y-DOTA-EB-TATE and PET as a substitute for ${ }^{90}$ Y-DOTA-EB-TATE for therapy. Dosimetric calculations revealed an increase of the tumor dose by a factor of 4 compared with the biodistribution without an albumin-binding functionality.

Similarly, Bandara et al. showed in a preclinical study that, in comparison to ${ }^{177} \mathrm{Lu}$-DOTATATE, ${ }^{177} \mathrm{Lu}$-DOTA-EB-TATE resulted in an increased tumor uptake over time, no unexpected accumulation, and efficient tumor control, despite similar activities (2).

Chen et al. applied ${ }^{177} \mathrm{Lu}$-DOTA-EB-TATE in humans and derived an increase in the tumor dose by a factor of 8 whereas kidney and bone marrow dose increased by a factor 3.2 and 18, respectively (3).

We investigated the labeling and the in vitro binding characteristics of ${ }^{68} \mathrm{Ga}-,{ }^{64} \mathrm{Cu}-,{ }^{177} \mathrm{Lu}$-, and ${ }^{90}$ Y-DOTA-EB-TATE in SSTRpositive HEK293-sstr 2 cells (4) in comparison to unmodified DOTA-TATE conjugates. The cells were plated in 6-well plates $24 \mathrm{~h}$ before addition of the radiolabeled DOTA-EB-TATE and DOTA-TATE conjugates $(200 \mathrm{kBq})$. Experiments were performed in $1 \mathrm{~mL}$ of standard cell culture medium at $37^{\circ} \mathrm{C}$ for 60 and $120 \mathrm{~min}$. At each time point, the cells were washed with phosphate-buffered saline and lysed with $0.3 \mathrm{M} \mathrm{NaOH}$. The uptake was determined in triplicate for each radionuclide and each time point and was expressed as a percentage of the total activity associated with 1 million cells. The labeling procedure was similar for all preparations, and the yields were more than $95 \%$. We found for DOTA-EB-TATE that after $1 \mathrm{~h}$ the cellular uptake was $9.1 \% \pm 0.69 \%, 2.0 \% \pm 0.12 \%, 4.9 \% \pm 0.26 \%$, and $4.6 \% \pm 0.21 \%$ and increased at $2 \mathrm{~h}$ to $15.6 \% \pm 0.51 \%, 4.3 \% \pm$ $0.18 \%, 9.0 \% \pm 0.71 \%$, and $8.1 \% \pm 0.12 \%$, respectively. In comparison, the bifunctional probes without the albumin-binding unit revealed a 1 -h uptake of $23.7 \% \pm 1.47 \%, 4.3 \% \pm 0.18 \%$, $8.7 \% \pm 0.90 \%$, and $7.2 \% \pm 1.09 \%$, which increased at $2 \mathrm{~h}$ to $27.3 \% \pm 1.66 \%, 5.5 \% \pm 0.36 \%, 12.6 \% \pm 1.71 \%$, and $10.2 \% \pm$ $0.18 \%$, respectively.

These data demonstrate that the modified radiotracer featured a lower initial uptake compared with the unmodified one, regardless which isotope was used. However, the incremental gain of the uptake within the second hour was comparable between both radiotracers. This demonstrates that the radioisotope strongly influences the uptake of the SSTR ligand. The highest diagnostic performance is expected from the radiopharmaceutical with the highest uptake, namely ${ }^{68} \mathrm{Ga}$-DOTATATE.

COPYRIGHT @ 2019 by the Society of Nuclear Medicine and Molecular Imaging.
As a consequence of the different uptake, only different isotopes of the same element (such as ${ }^{86} \mathrm{Y} /{ }^{90} \mathrm{Y}$ or ${ }^{64} \mathrm{Cu} /{ }^{67} \mathrm{Cu}$ ) can be used for assessment of biokinetic data, whereas theranostic "pairs" of isotopes (such as ${ }^{111} \mathrm{In} /{ }^{177} \mathrm{Lu}$ ) are not appropriate. No solely diagnostic isotope of lutetium is known. Therefore, the use of a low amount of radioactivity for qualitative and quantitative (e.g., dosimetry) imaging is an elegant approach that allows a subsequent therapeutic application (3).

\section{DISCLOSURE}

No potential conflict of interest relevant to this article was reported.

\section{ACKNOWLEDGMENTS}

We thank Dr. Xiaoyuan Chen, Bethesda, Maryland, for providing us DOTA-EB-TATE. The HEK293-sstr2 cells were a kind gift from Stephan Schulz, Jena, Germany.

\section{REFERENCES}

1. Tian R, Jacobson O, Niu G, et al. Evans blue attachment enhances somatostatin receptor subtype-2 imaging and radiotherapy. Theranostics. 2018;8: 735-745.

2. Bandara N, Jacobson O, Mpoy C, Chen X, Rogers BE. Novel structural modification based on Evans blue dye to improve pharmacokinetics of a somastostatin-receptorbased theranostic agent. Bioconjug Chem. 2018;29:2448-2454.

3. Zhang J, Wang H, Jacobson Weiss O, et al. Safety, pharmacokinetics and dosimetry of a long-acting radiolabeled somatostatin analogue ${ }^{177} \mathrm{Lu}-\mathrm{DOTA}-$ EB-TATE in patients with advanced metastatic neuroendocrine tumors. $\mathrm{J} \mathrm{Nucl}$ Med. 2018;59:1699-1705.

4. Lehmann A, Kliewer A, Schutz D, Nagel F, Stumm R, Schulz S. Carboxylterminal multi-site phosphorylation regulates internalization and desensitization of the human sst2 somatostatin receptor. Mol Cell Endocrinol. 2014;387:44-51.

Jörg Kotzerke*
Roswitha Runge
Anja Braune
Gerd Wunderlich
*Universitätsklinikum Dresden
Fetscherstrasse 74
01307 Dresden, Germany
E-mail: joerg.kotzerke@mailbox.tu-dresden.de

Published online Nov. 15, 2018.

DOI: 10.2967/jnumed.118.220707

\section{Reply: Different Radionuclides in DOTA-EB- TATE Effect Different Uptake in Somatostatin Receptor-Positive HEK293 Cells}

REPLY: We would like to thank Dr. Kotzerke and his colleagues for the important insights into the uptake of DOTAEB-TATE, an albumin-binding octreotate developed by us 